\title{
Validation of the Meaning in Life Questionnaire (MLQ) in a Greek Sample
}

\author{
Christos Pezirkianidis, Michael Galanakis, Irene Karakasidou, Anastasios Stalikas \\ Panteion University of Social and Political Sciences, Athens, Greece \\ Email: galanakismichael@hotmail.com
}

How to cite this paper: Pezirkianidis, C., Galanakis, M., Karakasidou, I., \& Stalikas, A. (2016). Validation of the Meaning in Life Questionnaire (MLQ) in a Greek Sample. Psychology, 7, 1518-1530. http://dx.doi.org/10.4236/psych.2016.713148

Received: April 6, 2016

Accepted: November 7, 2016

Published: November 10, 2016

Copyright $\odot 2016$ by authors and Scientific Research Publishing Inc. This work is licensed under the Creative Commons Attribution International License (CC BY 4.0).

http://creativecommons.org/licenses/by/4.0/

\begin{abstract}
Over the past few years, the concern for psychological well-being has emerged as one of the most crucial targets for social scientists and health professionals. One of the factors that contribute to psychological health is finding meaning in ones' life. Contemporary literature distinguishes between two types of meaning in life, namely 1) searching for meaning in life and 2) meaning in life achievement. The most prominent psychometric tool for the measurement of meaning in life is the Meaning in Life Questionnaire. The present study aimed at validating the MLQ in a sample of 6287 Greek adults. Exploratory factor analysis revealed two principal factors matching the factorial structure of the original validation. Results showed overall that all items have satisfactory psychometric properties. Further research and validation targets are discussed.
\end{abstract}

\section{Keywords}

Validation, Meaning in Life Questionnaire, Psychometric Properties, Greek Population

\section{Introduction}

In the last few years, the construct of meaning in life has received renewed attention, in conjunction with a growing focus on other positive variables (e.g. psychological strengths; Ryan \& Deci, 2001; Seligman \& Csikszentmihalyi, 2000). Inevitably, meaning in life is regarded as a positive variable, as an indicator of well-being (Ryff, 1989; Steger \& Frazier, 2005), a facilitator of adaptive coping (Park \& Folkman, 1997), or a marker of therapeutic growth (Crumbaugh \& Maholick, 1964; Frankl, 1965). Frankl (1963) emphasized the important connection between meaning in life and well-being. He suggested that the difficulty to find meaning in life is usually associated with emptiness and apa- 
thy, and could lead to several psychological problems. Recent studies have found that meaning in life is not only related with psychological health, but might also provide physical health benefits (Brassai, Piko, \& Steger, 2011).

In theory and research, two important dimensions of meaning in life have emerged (e.g., Crumbaugh, 1977; Steger, Frazier, Oishi, \& Kaler, 2006). The first concerns the degree to which individuals perceive their lives as significant and meaningful, referred to as "presence of meaning in life". The second refers to the degree to which people are engaged in a "search for meaning in life" (Steger, in press).

Research findings indicate that meaning in life is positively related to positive emotions, self-esteem, optimism, hope, happiness, curiosity, positive social interaction ( $\mathrm{Pe}$ zirkianidis, Stalikas, Efstathiou, \& Karakasidou, 2016; Reker \& Chamberlain, 2000; Steger \& Kashdan, 2007; Steger, Kawabata, Shimai, \& Otake, 2008; Steger \& Shin, 2010), life satisfaction (Bonebright, Clay, \& Ankermann, 2000), better control over life and higher work engagement (Lopez \& Snyder, 2011). Moreover, individuals with high levels of meaning in life tend to experience lower levels of negative effect, suicidal ideation and substance abuse (Steger, Kashdan, Sullivan, \& Lorentz, 2008; Steger \& Kashdan, 2007). The results of a recent Greek study showed that the presence of meaning in life has a negative correlation with experiencing negative emotions, depression, anxiety, stress and economic crisis effects (Pezirkianidis, Stalikas, Efstathiou, \& Karakasidou, 2016).

On the other hand, search for meaning in life is found to predict depression and anxiety levels (Steger, Mann, Michels, \& Cooper, 2009) and to be related to higher neuroticism (Steger et al., 2006), lower levels of happiness, life satisfaction (Park, Park, \& Peterson, 2010), well-being and higher levels of negative affect (Steger, Kawabata et al., 2008). It seems that searching for meaning in life has negative effects on human functioning, whereas already having meaning in life is connected to positive human functioning (Lopez \& Snyder, 2011). People who search for meaning and lack a sense of meaning in their lives, report the worst health and the highest anxiety levels (Steger, Mann et al., 2009). However, the search for meaning is crucially essential to the wellbeing process of the individual, since it can result in achieving meaning in life (Steger \& Kashdan, 2007).

Research results indicated that searching for and having meaning in life are negatively correlated (Steger et al., 2006). Nevertheless, a lot of people report that their lives are meaningful, simultaneously search for new or additional sources of meaning in their lives (Steger, Kawabata et al., 2008). Moreover, people, who find meaning during the search process, seem to report higher levels of life satisfaction than those who keep searching for meaning for a longer period of time (Steger \& Kashdan, 2007).

Concerning gender differences, Steger and colleagues (2009) found no significant differences between men and women with respect to presence or searching for meaning in life. Furthermore, they suggested that the presence score tends to be higher and search score tends to be lower among older participants.

The Meaning in Life Questionnaire (Steger et al., 2006) assesses two dimensions of 
meaning in life using 10 items rated on a seven-point scale from "Absolutely True" to "Absolutely Untrue." The Presence of Meaning subscale measures respondents' perception of the degree of meaning in their lives. The Search for Meaning subscale measures respondents' engagement in and motivation to spend effort in order to find meaning in their lives. In the construction study, the Cronbach's alpha coefficients ranged between .81 and .86 for Presence and .84 and .92 for Search. One-month test-retest reliability coefficients were .70 for Presence and .73 for Search. Other studies (see Schulenberg, Strack, \& Buchanan, 2011) also provide support for the reliability and test-retest reliability of MLQ scores. Scores on each MLQ subscale range from 5 to 35, with higher scores indicating higher Presence and Search. The MLQ is already validated in Spanish (Steger, Frazier et al., 2008), Chinese (Wang \& Dai, 2008) and Japanese (Shimai, Otake, \& Steger, in Press).

The purpose of this study was to validate the Meaning in Life Questionnaire in a sample of Greek citizens. In particular, we aim to provide data regarding inter-item correlations, means, standard deviations, variances, Cronbach's a and factorial structure as well as relationship with specific criteria as life satisfaction, psychological resilience, inspiration, hope, subjective happiness, depression, anxiety, stress, positive and negative emotions in terms of criterion validity.

\section{Method}

\section{Participants and Procedure}

The sample consisted of 6287 Greek adults (2052 men, 32.6\%, 3195 women, 50.8\% and 1040 missing, $16.5 \%$ ), aging from 18 to 83 years old. The mean age for the total sample was $M_{\text {age }}=37.49, S D=13.65$, for men $M_{\text {age }}=38.17, S D=13.89$ and for women $M_{\text {age }}=$ 37.09, $S D=13.18$. The majority of the participants were married (3038 married, $48.3 \%$, 2669 unmarried, 42.5\%, 401 divorced, 6.4\%, 125 widowers, 2\%, 54 missing, 0.8\%), employed (5059 employed, 80.5\%, 1172 unemployed, 18.6\%, 56 missing, 0.9\%), university graduates (2299 school graduates, 36.6\%, 721 university students, $11.5 \%, 2463$ university graduates, $39.2 \%, 758$ postgraduates, $12.1 \%, 46$ missing, $0.6 \%$ ).

The present data are a subset of a larger data bank of an ongoing longitudinal study, which started in 2008, examining the effects of the economic crisis on the psychological health of Greeks in relation to several variables including positive and negative emotions. The present data were collected during the years 2008 to 2014 with the help of undergraduate psychology students, who volunteered to administer the battery of tests. The volunteers were told that the purpose of the study was to examine the effects of the economic crisis on the well-being of Greeks and they were trained on the distribution, administration and collection of the questionnaires. Each student administered the battery of tests to 15 adult individuals among their social milieu. Every year approximately 100 students participated, resulting in the annual collection of approximately 1500 participants. Administration was done individually and was completed in approximately 20 minutes. The data were recorded on answer sheets and scanned using the $6^{\text {th }}$ Version of Remark Office OMR. 
In order to examine the criterion validity of the test, some participants also filled in other scales, which were used as criteria.

\section{Measures}

\subsection{Meaning in Life}

The Meaning in Life Questionnaire (MLQ; Steger, Frazier, Oishi, \& Kaler, 2006) is designed to measure the presence of meaning (how much respondents feel their lives have meaning) and the search for meaning in life (how much respondents strive to find meaning and understanding in their lives) using 10 items rated on a 7-point Likert-type scale ranging from 1 (Absolutely True) to 7 (Absolutely Untrue). The Presence of Meaning (e.g., "My life has a clear sense of purpose") and the Search for Meaning subscales (e.g., "I am looking for something that makes my life feel meaningful") consist of five items each. We used the Greek version of the instrument (Filippi \& Stalikas, 2012).

\subsection{Positive and Negative Emotions}

The mDES (Fredrickson et al., 2003) asks participants to recall the past two weeks and rate their strongest experience of each of 20 specific emotions on a 5-point Likert scale (1-Not At All to 5-Extremely). We used the Greek version of the instrument (mDES, Galanakis, Stalikas, Pezirkianidis, \& Karakasidou, 2016). The Greek mDES measures nine positive (joy, hope, love, contentment, pride, interest, gratitude, amusement and sexual desire), eight negative (anger, sadness, disgust, shame, guilt, contempt, embarrassment and fear) and three separate emotions (awe, sympathy and surprise). In our sample $(N=6160)$, the subscales demonstrated good internal consistency (Positive Emotions subscale, $\alpha=.86$, Negative Emotions subscale, $\alpha=.78$ ).

\subsection{Subjective Happiness}

The Greek version of Subjective Happiness Scale (SHS; Lyubomirsky \& Lepper, 1999; Avgoustaki, Dimitriadou, \& Stalikas, 2012) was used to examine the subjectivity of persons' global happiness using four items rated on a 7-point Likert scale with higher scores reflecting greater happiness (e.g., "Some people are generally very happy. They enjoy life regardless of what is going on, getting the most out of everything. To what extent does this characterization describe you?"). In our sample $(N=4119)$, the scale demonstrated good internal consistency $(\alpha=.76)$.

\subsection{Life Satisfaction}

The Satisfaction With Life Scale (SWLS; Diener, Emmons, Larsen, \& Griffin, 1985) examines the global assessment of a person's quality of life according to his/her chosen criteria using five items rated on a 7-point Likert scale ranging from "Strongly Disagree" to "Strongly Agree (e.g., "I am satisfied with my life"). We used the Greek version of the scale (Stalikas \& Lakioti, 2012), which demonstrated good internal consistency in our sample $(\alpha=.84 ; N=1294)$. 


\subsection{Inspiration}

The Inspiration Scale (IS; Thrash \& Elliot, 2003) measures the frequency and the intensity in which individuals feel inspired using eight items rated on a 7-point Likert scale. Four items examine the frequency of the inspiration on a scale ranging from "Never" to "Very Often" (e.g., "I experience inspiration. How often does this happen?") and four items measure the intensity of the inspiration on a scale ranging from "Not At All" to "Very Strongly" (e.g., "I am inspired to do something. How deeply or strongly in general?"). A total score can be also calculated. We used the Greek version of the instrument (Avgoustaki, Dimitriadou, \& Stalikas, 2012; $N=191$ ), which demonstrated high internal consistency $(\alpha=.95)$.

\subsection{Hope}

The Greek version of the Hope Scale (HS; Snyder et al., 1991; Moustaki \& Stalikas, 2012) was used to measure individuals' sense of successful goal-directed determination and planning of ways to meet goals using eight items rated on a 4-point Likert scale ranging from "Definitely False" to "Definitely True". The subscale of Agency intends to capture the extent to which participants feel successfully determined in meeting goals (e.g., "I energetically pursue my goals"), whereas the subscale of Pathways intends to capture the extent to which participants perceive that there are available, successful plans to meet goals (e.g., "There are lots of ways around any problem"). A total score can be computed. In our sample $(N=1715)$, the scale demonstrated good internal consistency $(\alpha=.86)$.

\subsection{Psychological Resilience}

The Greek version of the Connor-Davidson Resilience Scale (CD-RISC; Connor \& Davidson, 2003; Dimitriadou \& Stalikas, 2012) was used to measure individuals' stress coping ability and recovery from stress using 25 items rated on a 5-point Likert scale, with higher scores reflecting greater resilience (e.g., "Can handle unpleasant feelings"). The scale consists of five factors (personal competence, tolerance, acceptance of change, control and spiritual influences), but also, a total resilience score can be computed. In our sample $(N=4515)$, the scale demonstrated high internal consistency $(\alpha=.90)$.

\subsection{Psychological Health}

The Greek version of the Depression Anxiety and Stress Scale (DASS; Stalikas \& Flora, 2012; Lovibond \& Lovibond, 1995) was used to measure three related negative emotional states: depression (e.g., "I couldn't seem to experience any positive feeling at all"), anxiety (e.g., "I found myself in situations that made me so anxious I was most relieved when they ended") and tension/stress (e.g., "I found it difficult to relax"). Participants were asked to indicate the presence of 21 symptoms "over the previous week". Each item was rated from 1 (Did Not Apply to Me At All) to 4 (Applied to Me Very Much or Most of the Time). Each of the three subscales consisted of seven items. In our sample $(N=6113)$, the three subscales demonstrated high internal consistency ( $\alpha$ 
$=.91, .90$ and .89 , respectively).

\section{Results}

\subsection{Item Analysis}

We estimated variances, means, and standard deviations for the ten items of the scale in order to examine item quality and probability of dysfunctional items or polarization. According to the methodological rule we expected variances ranging from 1.5 to 3.0, indicative of a normal distribution regarding the given answers (reports of presence of meaning and search for meaning in life were rated on an anchored 7-point scale on which $1=$ Absolutely Untrue and $7=$ Absolutely True). Moreover, we were expecting means ranging from 3 to 5 also indicative of a normal distribution regarding the answers in the validation sample. Results shown in Table 1 indicate that all items have a normal distribution regarding the sample's answers.

Some concerns can be risen regarding item No.1 (I understand my life's meaning) and item No.7 (I am always searching for something that makes my life feel significant). These two items have a somewhat higher mean $(5.11,5.05)$ and a lower than anticipated variance $(1.99,1.71)$. However, it is not unlikely to have such higher scores in non-clinical population like ours. The reliability analysis and factor analysis that follow also indicate that these two items do not pose a threat to the psychometric properties of the scale. In light of this we have decided to keep these items in the Greek version of the test.

\subsection{Item Inter-Correlations}

To further examine item quality we carried out a correlational analysis between all scale items. Since the scale measures two different dimensions, namely presence of meaning and search for meaning, we performed two separate correlational analyses, one for the

Table 1. Means, standard deviations and variances of the MLQ items $(1=$ absolutely true to $5=$ absolutely untrue; $N=6287$ ).

\begin{tabular}{cccc}
\hline Items & Mean & SD & Variance \\
\hline 1. I understand my life's meaning. & 5.11 & 1.41 & 1.99 \\
2. I am looking for something that makes my life feel meaningful. & 4.91 & 1.74 & 3.04 \\
3. I am always looking to find my life's purpose. & 4.72 & 1.72 & 2.95 \\
4. My life has a clear sense of purpose. & 4.98 & 1.52 & 2.32 \\
5. I have a good sense of what makes my life meaningful. & 5.25 & 1.43 & 2.05 \\
6. I have discovered a satisfying life purpose. & 5.02 & 1.43 & 2.05 \\
7. I am always searching for something that makes & & 1.54 & 1.71 \\
my life feel significant. & 5.05 & 1.71 & 2.92
\end{tabular}

Note: Item No 9 (My life has no clear purpose) is included to the analysis after being reversed. 
Presence subscale items and one for the Search subscale items. According to the methodological rule we were expecting to find positive statistical significant correlations between the items of each subscale ranging from .10 to .60 . This particular strength and direction of the correlation is indicative of items that measure the same variable and are complementary to one another regarding the factor variable. Negative correlations are indicative of opposite variables measurement, while null correlations are indicative of irrelevancy to the main variable. Extremely high correlations $(r>.60)$ are indicative of items that probably measure the exact same thing and therefore one of them could be omitted without losing any psychometric properties. Table 2 and Table 3 show the results of the analyses.

According to the results shown in Table 2 and Table 3 most inter-item correlations in the two MLQ subscales were positive and statistically significant $(p<.001)$, ranging from $r=.32$ to .60 as expected. Higher inter-correlations were found between items 5 and $6(r=.67)$, which will be evaluated combined with the reliability results in order to decide whether one of the two items is not needed in the questionnaire. The above findings are indicative of adequate construct validity.

Table 2. Inter-item correlations between the items of the presence of meaning subscale $(N=$ 6287).

\begin{tabular}{|c|c|c|c|c|c|}
\hline & 1. & 4. & 5. & 6. & 9. \\
\hline 1. I understand my life's meaning. & - & & & & \\
\hline 4. My life has a clear sense of purpose. & .56 & - & & & \\
\hline $\begin{array}{l}\text { 5. I have a good sense of what makes my life } \\
\text { meaningful. }\end{array}$ & .56 & .58 & - & & \\
\hline 6. I have discovered a satisfying life purpose. & .54 & .58 & .67 & - & \\
\hline 9. My life has no clear purpose. & .38 & .44 & .37 & .41 & - \\
\hline \multicolumn{6}{|c|}{$\begin{array}{l}{ }^{*} \text { Every correlation is significant at the } .001 \text { level. Note. Item No } 9 \text { (My life has no clear purpose) is included to } \\
\text { analysis after being reversed. }\end{array}$} \\
\hline & 2. & 3. & 7. & 8. & 10. \\
\hline $\begin{array}{l}\text { 2. I am looking for something that } \\
\text { makes my life feel meaningful. }\end{array}$ & - & & & & \\
\hline $\begin{array}{l}\text { 3. I am always looking to find my } \\
\text { life's purpose. }\end{array}$ & .60 & - & & & \\
\hline $\begin{array}{l}\text { 7. I am always searching for something that } \\
\text { makes my life feel significant. }\end{array}$ & .47 & .52 & - & & \\
\hline $\begin{array}{l}\text { 8. I am seeking a purpose or } \\
\text { mission for my life. }\end{array}$ & .50 & .55 & .58 & - & \\
\hline 10. I am seeking for meaning in my life. & .45 & .43 & .32 & .49 & - \\
\hline
\end{tabular}

${ }^{\star}$ Every correlation is significant at the .001 level. 


\subsection{Reliability}

We estimated the scale reliability using the Cronbach alpha index as well as split half reliability measures. According to the analysis, the MLQ can be used as a reliable tool for the assessment of presence and search for meaning in the Greek population. Specifically, the Cronbach Alpha index for the scale was $a=.76$, for the Presence subscale was $a=.83$ and for the Search subscale was $a=.82$. The split half reliability index for the ten MLQ items was Spearman-Brown Coefficient (equal and unequal length) $=.66$ and Guttman Coefficient $=.65$. Higher split half indexes were found for the Presence subscale (Spearman-Brown Coefficient $=.82$, Guttman Coefficient $=.79$ ) and the Search subscale (Spearman-Brown Coefficient $=.79$, Guttman Coefficient $=.77$ ). Further item analysis exploring the possibility to strengthen the scale reliability if any of the items was deleted gave negative results. Hypothetical deletion of items leads to reliability decrease. In light of the item analysis results (reliability if item deleted) we decided to maintain the ten initial scale items.

\subsection{Factor Analysis}

In order to examine the factorial structure of the scale we proceeded to Exploratory Factor Analysis. Based on the factorial structure of the original version of the test we expected different factor loadings for presence of meaning and search for meaning in life items. Table 4 shows the results of the exploratory factor analysis.

According to the factor analysis and the Kaiser criterion (eigenvalues greater than 1) there seem to be two principal factors in the scale which explain $62.06 \%$ of the variable

Table 4. MLQ Item loadings per factor.

\begin{tabular}{|c|c|c|c|}
\hline Item No. & Items & Factor 1 & Factor 2 \\
\hline 6 & I have discovered a satisfying life purpose. & .791 & \\
\hline 5 & I have a good sense of what makes my life meaningful. & .779 & \\
\hline 4 & My life has a clear sense of purpose. & .748 & \\
\hline 1 & I understand my life's meaning. & .700 & \\
\hline 9 & My life has no clear purpose. & .553 & \\
\hline 8 & I am seeking a purpose or mission for my life. & & .761 \\
\hline 3 & I am always looking to find my life's purpose. & & .751 \\
\hline 2 & $\begin{array}{l}\text { I am looking for something that } \\
\text { makes my life feel meaningful. }\end{array}$ & & .717 \\
\hline 7 & $\begin{array}{l}\text { I am always searching for something that } \\
\text { makes my life feel significant. }\end{array}$ & & .666 \\
\hline \multirow[t]{4}{*}{10} & I am seeking for meaning in my life. & & 630 \\
\hline & Eigenvalues & 3.438 & 2.768 \\
\hline & Variance explained & $34.38 \%$ & $27.68 \%$ \\
\hline & Total variance explained & $62.06 \%$ & \\
\hline
\end{tabular}

Extraction method: Principal Axis Factoring; Rotation method: Varimax. 
variance. The same conclusion can also be drawn from the scree plot and the Monte Carlo PCA for Parallel Analysis (two of the MLQ factors' eigenvalues are greater than random eigenvalues). The first factor consists of five presence of meaning items, and the second factor consists of five search for meaning items. No double loadings were found. The factor analysis confirms the original factorial structure of the scale.

\subsection{Criterion Validity Analysis}

In order to further examine the validity of the scale we used as criteria other variables. We hypothesized that presence of meaning in life score would correlate negatively to Negative Emotions (NE), Stress (STR), Depression (DEP) and Anxiety (ANX), and positively to Life Satisfaction (LS), Psychological Resilience (RES), Inspiration (IN), Hope (HO), Positive Emotions (PE) and Subjective Happiness (SH). On the other hand, it was hypothesized that the Search subscale would show low positive correlations with the aforementioned criteria, since searching for meaning in life in some cases correlate positively with psychopathology and experiencing negative emotions, and in other cases can lead to positive outcomes. Results are presented in Table 5.

Results show that both Presence and Search subscales have satisfactory criterion validity. As hypothesized, the Presence subscale showed: a) low negative correlation to depression $(r=-.27, p<0.001, N=6113)$, anxiety $(r=-.18, p<.001, N=6113)$, stress $(r=-.15, p<.001, N=6113)$ and experiencing of negative emotions $(r=-.20, p<.001$, $N=6160)$, b) low positive correlation to inspiration $(r=.29, p<.001, N=191)$, subjective happiness $(r=.22, p<.001, N=4119)$ and experiencing of positive emotions $(r=.28$, $p<.001, N=6,160)$, and c) moderate positive correlation to life satisfaction $(r=.49$, $p<.001, N=1294)$, psychological resilience $(r=.52, p<.001, N=4515)$ and hope $(r=.50$, $p<.001, N=1715)$. On the other hand, the Search subscale showed low positive correlation to almost every criterion, as hypothesized. More specifically, it was positively correlated to life satisfaction $(r=.08, p<.01, N=1294)$, psychological resilience $(r=.22$, $p<.001, N=4515)$, inspiration $(r=.24, p<.001, N=191)$, hope $(r=.20, p<.001, N=$ 1715), depression $(r=.05, p<.001, N=6113)$, anxiety $(r=.06, p<.001, N=6113)$, stress $(r=.09, p<.001, N=6113)$, experiencing of positive $(r=.12, p<.001, N=6160)$ and negative emotions $(r=.06, p<.001, N=6160)$. No correlation found between Search subscale and subjective happiness.

\subsection{Norms}

In order to help mental health professionals to interpret the scores of the subscales of

Table 5. Criterion validity of the MLQ (criteria: Life satisfaction, psychological resilience, inspiration, hope, subjective happiness, depression, anxiety, stress, positive and negative emotions).

\begin{tabular}{ccccccccccc}
\hline & LS & RES & IN & HO & SH & DEP & ANX & STR & PE & NE \\
\hline Presence & $.49^{* *}$ & $.52^{* *}$ & $.29^{* *}$ & $.50^{\star *}$ & $.22^{* *}$ & $-.27^{* *}$ & $-.18^{* *}$ & $-.15^{* *}$ & $.28^{* *}$ & $-.20^{* *}$ \\
Search & $.08^{*}$ & $.22^{\star *}$ & $.24^{* *}$ & $.20^{* *}$ & .00 & $.05^{* *}$ & $.06^{* *}$ & $.09^{* *}$ & $.12^{* *}$ & $.06^{* *}$ \\
$\mathrm{~N}$ & 1294 & 4515 & 191 & 1715 & 4119 & 6113 & 6113 & 6113 & 6160 & 6160 \\
\hline
\end{tabular}

${ }^{*} p<.01,{ }^{* *} p<.001$. 
MLQ, we calculated the normalized scores using the Stanscore 4 program. In Table 6 professionals and researchers can match the raw score of the two subscales to a Sten Score ranging from 1 to 10 so as to compare the individual's score with the norm.

\section{Discussion}

This study provides empirical support for the reliability and validity of the Greek version of the Meaning in Life Questionnaire (Steger et al., 2006). Adaptation was based on data collected from 6287 individuals residing in urban areas, using common component analysis. Results showed that the scale's items have satisfactory psychometric qualities. Mean scores ranged from 4.17 to 5.25 while variance ranged from 1.71 to 4.25 per item. Mean scores per item in relation to standard deviations and variances are indicative of normal distribution as far as participants' answers are concerned. Some concerns can be risen regarding item No.1 (I understand my life's meaning) and item No.7 (I am always searching for something that makes my life feel significant). These two items have a somewhat higher mean $(5.11,5.05)$ and a lower than anticipated variance $(1.99,1.71)$. One possible explanation for the means and variances in these two items may be that they represent common pursuits and beliefs that are often experienced in non-clinical populations. Moreover, these two items were useful to the questionnaire's reliability and factorial structure.

To further examine item quality we estimated item inter-correlations expecting positive statistically significant correlations ranging from 0.1 to 0.6 . The analysis showed that correlations between all items ranged from $r=.38$ to $r=.67$ as expected. This finding is indicative of adequate construct validity.

As far as reliability is concerned, the scale has satisfactory reliability. Specifically, the Cronbach Alpha index for the scale was $a=.76$, for the Presence subscale was $a=.83$ and for the Search subscale was $a=.82$. The split half reliability index for the ten MLQ items was Spearman-Brown Coefficient (equal and unequal length) $=.66$ and Guttman Coefficient $=.65$. Higher split half indexes were found for the Presence subscale

Table 6. Norms of the presence and search subscales of the MLQ.

\begin{tabular}{cccc}
\hline $\begin{array}{c}\text { Presence of meaning } \\
\text { raw score range }\end{array}$ & Sten equivalent & Description & $\begin{array}{c}\text { Search for meaning } \\
\text { raw score range }\end{array}$ \\
\hline 5 to 13 & 1 & Very Low & 5 to 7 \\
14 to 16 & 2 & Low & 8 to 11 \\
17 to 19 & 3 & Low & 12 to 16 \\
20 to 22 & 4 & Medium & 17 to 20 \\
23 to 26 & 5 & Medium & 21 to 24 \\
27 to 29 & 6 & Medium & 25 to 27 \\
30 to 31 & 7 & Medium & 28 to 30 \\
32 to 33 & 8 & High & 31 to 32 \\
34 & 9 & High & 33 to 34 \\
35 & 10 & Very High & 35 \\
\hline
\end{tabular}


(Spearman-Brown Coefficient $=.82$, Guttman Coefficient $=.79)$ and the Search subscale (Spearman-Brown Coefficient $=.79$, Guttman Coefficient $=.77$ ). Moreover item analysis showed that possible deletion of any number of items could not increase the reliability level of the questionnaire.

Factorial structure of the Meaning in Life Questionnaire was examined through exploratory factor analysis. According to the factor analysis and the Kaiser criterion (eigenvalues greater than 1) there seem to be two principal factors which explain $62.06 \%$ of the variable variance. The same conclusion can also be drawn from the scree plot and the Monte Carlo PCA for Parallel Analysis (two of the MLQ factors' eigenvalues are greater than random eigenvalues). The first factor consists of five presence of meaning items, and the second factor consists of five search for meaning items. No double loadings were found. The factor analysis confirms the original factorial structure of the scale.

Additionally, we provided further empirical support regarding the validity of the questionnaire using as criteria specific variables based on the literature mentioned in the introduction section. In particular we used the following variables as criteria: Negative Emotions (NE), Stress (STR), Depression (DEP), Anxiety (ANX), Life Satisfaction (LS), Psychological Resilience (RES), Inspiration (IN), Hope (HO), Positive Emotions (PE) and Subjective Happiness (SH). Results show that both Presence and Search subscales have satisfactory criterion validity.

Regarding the limitations of our study, we should mention that reliability indexes were not calculated using test-retest methodology but only simultaneously using the Cronbach Alpha and Split Half Indexes. Moreover, all criteria validity measures were concurrent while we could also estimate validity measures over a period of time and regarding future results.

The study provides useful insights regarding the utilization of the Meaning in Life Questionnaire in future studies in Greek speaking populations and it could enhance positive psychology research in all levels.

\section{Conclusion}

We strongly believe that future research regarding the validation of the Meaning in Life Questionnaire in the Greek population could focus in more specific positive psychology measures while also exploring the cultural differences between different populations regarding meaning in Life. In all, the Meaning in Life Questionnaire-Greek Version can be used as a reliable and valid psychometric tool for the measurement of Meaning in Life in the Greek population.

\section{References}

Bonebright, C. A., Clay, D. L., \& Ankermann, R. D. (2000). The Relationship of Workaholism with Work-Life Conflict, Life Satisfaction, and Purpose in Life. Journal of Counseling Psychology, 47, 469-477. http://dx.doi.org/10.1037/0022-0167.47.4.469

Brassai, L., Piko, B. F., \& Steger, M. F. (2011). Meaning in Life: Is It a Protective Factor for Adolescents' Psychological Health? International Journal of Behavioral Medicine, 18, 44-51. 
http://dx.doi.org/10.1007/s12529-010-9089-6

Crumbaugh, J. C. (1977). The Seeking of Noetic Goals Test (SONG): A Complementary Scale to the Purpose in Life Test (PIL). Journal of Clinical Psychology, 33, 900-907. http://dx.doi.org/10.1002/1097-4679(197707)33:3<900::AID-JCLP2270330362>3.0.CO;2-8

Frankl, V. E. (1963). Man's Search for Meaning: An Introduction to Logotherapy. New York: Washington Square Press.

Frankl, V. E. (1965). The Doctor and the Soul: From Psychotherapy to Logotherapy. New York: Vintage Books.

Lopez, S. J., \& Snyder C. R. (2011). The Oxford Handbook of Positive Psychology (2nd ed.). New York: Oxford University Press.

Park, C. L., \& Folkman, S. (1997). Meaning in the Context of Stress and Coping. Review of General Psychology, 30, 115-144. http://dx.doi.org/10.1037/1089-2680.1.2.115

Park, N., Park, M., \& Peterson, C. (2010). When Is Search for Meaning Related to Life Satisfaction? Applied Psychology: Health and Wellbeing, 2, 1-13. http://dx.doi.org/10.1111/j.1758-0854.2009.01024.x

Pezirkianidis, C., Stalikas, A., Efstathiou, E., \& Karakasidou, E. (2016). The Relationship between Meaning in Life, Emotions and Psychological Illness: The Moderating Role of the Effects of the Economic Crisis. The European Journal of Counseling Psychology, 4, 1-24. http://dx.doi.org/10.5964/ejcop.v4i1.75

Reker, G. T., \& Chamberlain, K. (2000). Exploring Existential Meaning: Optimizing Human Development across the Life Span. Thousand Oaks, CA: Sage Publication.

Ryan, R. M., \& Deci, E. L. (2001). On Happiness and Human Potentials: A Review of Research on Hedonic and Eudaimonic Well-Being. Annual Review of Psychology, 52, 141-166. http://dx.doi.org/10.1146/annurev.psych.52.1.141

Ryff, C. D. (1989). Happiness Is Everything, or Is It? Explorations on the Meaning of Psychological Well-Being. Journal of Personality and Social Psychology, 57, 1069-1081.

http://dx.doi.org/10.1037/0022-3514.57.6.1069

Seligman, M. E. P., \& Csikszentmihalyi, M. (2000). Positive Psychology: An Introduction. American Psychologist, 55, 5-14. http://dx.doi.org/10.1037/0003-066X.55.1.5

Schulenberg, S. E., Strack, K. E., \& Buchanan, E. M. (2011). The Meaning in Life Questionnaire: Psychometric Properties with Individuals with Serious Mental Illness in an Inpatient Setting. Journal of Clinical Psychology, 67, 1210-1219. http://dx.doi.org/10.1002/jclp.20841

Shimai, S., Otake, K., \& Steger, M.F. (In Press). The Japanese Meaning in Life Questionnaire.

Steger, M. F., Frazier, P., Oishi, S., \& Kaler, M. (2006). The Meaning in Life Questionnaire. Assessing the Presence of and Search for Meaning in Life. Journal of Counseling Psychology, 53, 80-93. http://dx.doi.org/10.1037/0022-0167.53.1.80

Steger, M. F., \& Kashdan, T. B. (2007). Stability and Specificity of Meaning in Life and Life Satisfaction over One Year. Journal of Happiness Studies, 8, 161-179.

http://dx.doi.org/10.1007/s10902-006-9011-8

Steger, M. F., Kawabata, Y., Shimai, S., \& Otake, K. (2008). The Meaningful Life in Japan and the United States: Levels and Correlates of Meaning in Life. Journal of Research in Personality, 42, 660-678. http://dx.doi.org/10.1016/j.jrp.2007.09.003

Steger, M. F., Mann, J. R., Michels, P., \& Cooper, T. C. (2009). Meaning in Life, Anxiety, Depression, and General Health among Smoking Cessation Patients. Journal of Psychosomatic Research, 67, 353-358. http://dx.doi.org/10.1016/j.jpsychores.2009.02.006

Steger, M. F., \& Shin, J. Y. (2010). The Relevance of the Meaning in Life Questionnaire to Thera- 
peutic Practice: A Look at the Initial Evidence. The International Forum for Logotherapy, 33, 95-104.

http://www.michaelfsteger.com/wp-content/uploads/2012/08/Steger-Shin-IFL-2010.pdf

Wang, M. C., \& Dai, X. Y. (2008). Chinese Meaning in Life Questionnaire Revised in College Students and Its Reliability and Validity Test. Chinese Journal of Clinical Psychology, 16, 459461.

Submit or recommend next manuscript to SCIRP and we will provide best service for you:

Accepting pre-submission inquiries through Email, Facebook, LinkedIn, Twitter, etc. A wide selection of journals (inclusive of 9 subjects, more than 200 journals)

Providing 24-hour high-quality service User-friendly online submission system

Fair and swift peer-review system Efficient typesetting and proofreading procedure

Display of the result of downloads and visits, as well as the number of cited articles Maximum dissemination of your research work

Submit your manuscript at: http://papersubmission.scirp.org/

Or contact psych@scirp.org 